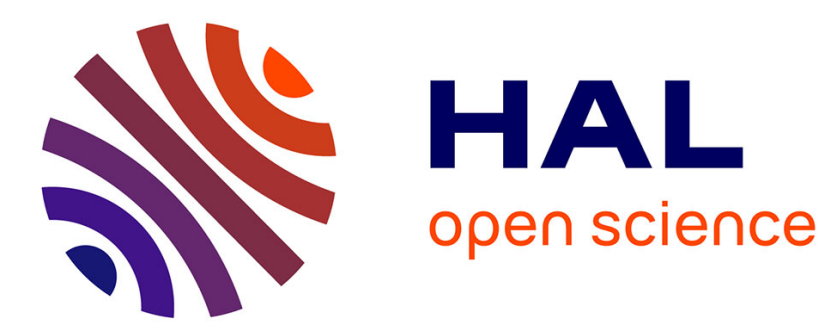

\title{
Extension du domaine de l'entrepreneurialité dans la pratique du vide-dressing
}

Elodie Juge, Isabelle Collin-Lachaud, Dominique Roux

\section{To cite this version:}

Elodie Juge, Isabelle Collin-Lachaud, Dominique Roux. Extension du domaine de l'entrepreneurialité dans la pratique du vide-dressing. Revue Française de Gestion, 2019, 45 (284), pp.31-49. 10.3166/rfg.2019.00372 . hal-03266096

\section{HAL Id: hal-03266096 https://hal.science/hal-03266096}

Submitted on 21 Jun 2021

HAL is a multi-disciplinary open access archive for the deposit and dissemination of scientific research documents, whether they are published or not. The documents may come from teaching and research institutions in France or abroad, or from public or private research centers.
L'archive ouverte pluridisciplinaire HAL, est destinée au dépôt et à la diffusion de documents scientifiques de niveau recherche, publiés ou non, émanant des établissements d'enseignement et de recherche français ou étrangers, des laboratoires publics ou privés. 
Extension du domaine de l'entrepreneurialité dans la pratique du vide-dressing

Elodie Juge, Isabelle Collin-Lachaud et Dominique Roux

Elodie Juge, PhD (Univ. Lille, ULR LUMEN, France)

IAE Lille University School of Management, Avenue du Peuple Belge, 59000 Lille

elodie.juge@univ-lille.fr

Isabelle Collin-Lachaud, Professor (Univ. Lille, ULR LUMEN, France)

IAE Lille University School of Management, Avenue du Peuple Belge, 59000 Lille isabelle.collin-lachaud@univ-lille.fr

Dominique Roux, Professor (Université de Reims Champagne, Ardenne, Laboratoire REGARDS, EA 6292)

Université de Reims Champagne, Ardenne, Bâtiment Recherche

BP 30, 57 rue Pierre Taittinger

51097 Reims, France

dominique.roux@univ-reims.fr

Pour citer l'article : Elodie Juge, Isabelle Collin-Lachaud et Dominique Roux (2019), Extension du domaine de l'entrepreneurialité dans la pratique du vide-dressing Revue Française de Gestion, vol. 45 n $^{\circ} 284$ p.31-49 


\title{
Extension du domaine de l'entrepreneurialité dans la pratique du vide-dressing
}

\section{Résumé}

Cette recherche éclaire la manière dont les nouveaux dispositifs sociotechniques (plateformes, applications mobiles...) remodèlent les échanges, tissent de nouvelles relations avec leurs usagers, transforment les rapports humains et modifient le rôle du consommateur dans la pratique du vide-dressing. Mobilisant une approche qualitative multi-méthodes sur une période de six années, cette recherche révèle à la fois les dimensions entrepreneuriales de la pratique et la «post-socialité » qu'elle participe à construire.

Mots-clés : économie collaborative, échanges pair à pair, consommateur-entrepreneur, (post)-socialité, objet épistémique.

\section{Extension of the domain of entrepreneurship: The "shop my closet" case}

\begin{abstract}
This research sheds light on the way by which new sociotechnical devices (platforms, mobile applications, etc.) reshape exchanges, alter the traditional sociality, weave new relationships with their users and finally alter the role of the consumer in the "shop my closet" practice. By mobilizing a multi-method qualitative approach over a six-year period, this research reveals both the entrepreneurial dimensions of the practice and the "post-sociality" that it participates to sketch.
\end{abstract}

Keywords: Collaborative economy, peer-to-peer exchanges, consumer/entrepreneur, (post-) sociality, epistemic object. 
L'essor rapide et important de l'économie collaborative - appelée encore économie du partage (Belk, 2010) - a profondément modifié les modes de production, de consommation et de distribution. Au sein de ce paysage, les plateformes jouent un rôle essentiel dans la fluidification des échanges (Benavent, 2016), offrant aux producteurs et aux distributeurs un accès direct à leurs clients et permettant à des particuliers d'acheter et de vendre à d'autres particuliers (Hamari, Sjöklint et Ukkonen, 2015). En dépit des débats sur le périmètre, les frontières et les trajectoires possibles de l'économie collaborative (Acquier, Carbone et Massé, 2017; Decrop, 2017 ; Herbert et CollinLachaud, 2016), un consensus existe sur les formes d'empowerment qu'elle offre aux consommateurs (Ertz, Durif et Arcand, 2016), leur permettant de remettre leurs biens en circulation (par le don, le troc, ou la revente), de les mutualiser de manière séquentielle ou simultanée (via le prêt, la location ou le partage), ou de partager des connaissances, des services ou des modes de vie participatifs autour de ces objets (Botsman et Rogers, 2011). De nombreux aspects de l'économie collaborative ont déjà été investigués ces dernières années, notamment son orientation économique versus altruiste (Herbert et Collin-Lachaud, 2016), les formes de socialité qui s'y déploient (Borel, Guillard et Roux, 2017), les motivations critiques qui animent ses usagers (Ozanne et Ballantine, 2010), mais aussi la compensation morale qui conduit des sujets sensibles à l'environnement, paradoxalement, à surconsommer (Parguel, Lunardo et Benoit-Moreau, 2017). Alors que la littérature couvre un large spectre de pratiques collaboratives, la revente vestimentaire est comparativement peu investiguée. Le vêtement est en effet un bien particulier qui touche au corps - contaminé et contaminant (Roux et Korchia, 2006) -, de fait moins réputé s'échanger entre étrangers qu'entre pairs (Clarke 2000 ; Lurie, 1981). Pederson et Netter (2015) montrent toutefois que des jeunes femmes désargentées, sensibles à l'apparence ou aux enjeux écologiques sont adeptes de la revente vestimentaire alors même qu'elles ne se connaissent pas. Par ailleurs, sous l'angle strictement économique et comparé à d'autres pratiques d'achat/vente, le vide-dressing touche un produit de consommation de masse, et non un bien d'investissement lourd plus facile à valoriser (comme l'immobilier ou la voiture), dont la valeur unitaire peut toutefois se révéler élevée comparée à son faible taux d'usage (Pike, 2016). De fait, l'engouement récent pour cette pratique en fait un phénomène paradoxal et intéressant à étudier en raison de ses spécificités, notamment sa capacité à revaloriser des produits intimes, à forte rotation, soumis aux effets de mode de la fast-fashion, mais aussi des produits possédés en nombre que l'exiguité des logements contraint à évacuer pour en posséder d'autres.

Par ailleurs, ces échanges pair à pair ne questionnent pas seulement les soubassements idéologiques qui les animent (Acquier, Carbone et Massé, 2017), ni non plus exclusivement le devenir de la production et de la distribution conventionnelles, c'est-à-dire le modèle linéaire production-distribution-consommation (Bauhain-Roux et Guiot, 2001) dans lequel le 
consommateur reste cantonné à un rôle passif d'acheteur, d'utilisateur et de destructeur de ressources (Ertz, Durif et Arcand, 2016). Plus fondamentalement, notre étude montre que ces pratiques s'inscrivent dans une «forme entreprise » qui soutient la rationalité néolibérale depuis le début du $20^{\text {ème }}$ siècle (Foucault, 2004, p.154). Par «forme entreprise », on entend la dynamique concurrentielle qui régit toutes les sphères de la vie sociale, poussant l'individu à devenir «entrepreneur de lui-même », c'est-à-dire «étant à lui-même son propre capital, étant pour lui-même son propre producteur, étant pour lui-même la source de [ses] revenus » (Foucault, 2004, p. 221). La diffusion de cet esprit d'entreprise va également de pair avec le fait que l'individu-entrepreneur doit investir de manière continue dans le développement de son capital humain pour espérer faire fructifier ses acquis (Becker, 1964). Qu'il s'agisse de l'auto-entreprenariat (Abdelnour, 2016) ou de la revente de ses biens personnels (Lemaitre et De Barnier, 2015), l'individu développe de nouvelles compétences qui l'apparentent à un professionnel. Plus encore, le développement des modes d'intermédiation, numériques ou non, contribue à produire de nouvelles relations entre les individus et les dispositifs qui soutiennent ces activités. Ceux-ci ne sont pas seulement des instruments au service de ces pratiques, mais les supports relationnels privilégiés avec lesquels les individus interagissent, ce que Knorr Cetina (1997), à la suite de Rheinberger (1992), nomme « objets épistémiques ». Ouverts, matériellement évolutifs, en perpétuelle transformation, les objets épistémiques confrontent les individus à un apprentissage constant et toujours plus complexe à mesure qu'ils tentent de les maîtriser. Par un cycle continu de découvertes qui lui laisse espérer qu'il progresse dans leur maniement, l'homo oeconomicus contemporain développe ainsi avec ces objets des relations d'attachement « de manière intime et quasi sociale » (Zwick et Dholakia, 2006). De fait, l'entrepreneuralité se joue aussi sur l'arrière-plan d'une «société de la connaissance » (Stehr, 1994) qui fabrique une socialité nouvelle centrée sur les objets.

Notre recherche vise à examiner la dimension entrepreneuriale d'une activité marchande pair à pair et l'influence que les dispositifs sociotechniques exercent sur les rapports humains (Knorr Cetina, 1997). La pratique du vide-dressing (achat/vente de vêtements et d'accessoires d'occasion entre particuliers) nous permet d'explorer la nature des relations qui se nouent entre les pratiquantes et leurs clientes, mais aussi entre les pratiquantes et les dispositifs sociotechniques par lesquels elles apprennent, comprennent et accumulent des savoirs et savoir-faire marchands. Ces dispositifs comportent par ailleurs une double modalité : une version en face-à-face lorsque les pratiquantes organisent des échanges à leur domicile ou que des intermédiaires (tels Violette Sauvage ou Secret de Commode) se chargent de leur mise en place ; une version en ligne qui s'exerce de manière complémentaire 
via des réseaux-sociaux (Facebook), des plateformes généralistes (ebay ou leboncoin) ou encore via des sites spécialisés comme Vinted ou VestiaireCollective.

Par le biais d'une étude qualitative multi-méthodes à caractère ethnographique menée sur une longue période (six années), notre recherche met au jour que la pratique d'achat/vente de vêtements et d'accessoires d'occasion, à la fois en face-à-face et en ligne, constitue pour les pratiquantes du vide-dressing une activité entrepreneuriale et un nouvel espace d'apprentissage qui transforme les socialités primaires (de l'interconnaissance, Borel, 2011). Après avoir présenté le cadre conceptuel de notre recherche ainsi que son dispositif méthodologique, les résultats sont détaillés, puis discutés.

\section{I - DU POST-CONSOMMATEUR ENTREPRENEUR...}

Les «figures » du consommateur post-moderne ont considérablement évolué (Cova et Cova, 2009), dévoilant un individu pluriel, tour à tour client, usager, citoyen, co-producteurtravailleur (Dujarier, 2008) - ou prosumer (Tofler, 1980) - et marketeur de lui-même, de son image ou de ses biens. En réalité, la créativité, l'esprit d'initiative et le travail productif du consommateur ont toujours existé (Ritzer et Jurgenson, 2010). Mais si ces caractéristiques paraissent aujourd'hui mises en avant, c'est par un effet de miroir des discours et des dispositifs par lequel un travail institutionnel «produit» ce «nouveau consommateur» (Cova et Cova, 2009). L'idéologie collaborative participe de la même manière à véhiculer une représentation particulière du consommateur : celle d'un individu réengagé par ces nouvelles pratiques dans les socialités anciennes du don et du partage que le marché aurait progressivement sapées. L'individu, libre et affranchi des systèmes traditionnels, serait capable de se «bricoler » des activités sur mesure, jouant alternativement le rôle d'acheteur et celui de vendeur (Lemaitre et De Barnier, 2015), et faisant de la consommation un loisir autant qu'une profession (chauffeur Uber par exemple). L'essor des échanges pair à pair peut être envisagé comme un rééquilibrage du rapport de force entre consommateurs et organisations marchandes, tout en soulignant parallèlement que les dispositifs sociotechniques qui soutiennent ces pratiques servent à produire des ressources connaissances, données, travail réel - dont se nourrit, souvent à peu de frais, un capitalisme d'un genre nouveau (Benavent, 2016 ; Ritzer et Jurgenson, 2010).

Il faut en réalité remonter historiquement à la source de la gouvernementalité néolibérale pour mieux situer l'origine de l'entrepreuneurialité à l'œuvre dans ces nouvelles pratiques, dont la consommation collaborative est le récent avatar. Si Schumpeter (1990 [1947]) fait de 
l'entrepreneur la figure emblématique de la transformation créative de l'économie et le moteur de la dynamique du capitalisme, la rationalité néolibérale l'a généralisée à l'ensemble des agents économiques. Comme le soulignent Dardot et Laval (2009, p. 226), la rationalité néolibérale conçoit le marché comme «un processus d'autoformation du sujet économique, comme un processus subjectif auto-éducateur et auto-disciplinaire par lequel l'individu apprend à se conduire ». L'économie devient ainsi, de manière extensive, « une science du choix » et « de tous les genres de l'agir humain »(Von Mises, 1985). Ni laissez-fairiste, ni interventionniste sur le plan politique, la gouvernementalité néolibérale indexe de facto toute la politique de la société sur le gouvernement de soi de l'individu, lui-même inspiré du modèle de l'entreprise. L'individu-entrepreneur est censé diriger sa vie comme il gèrerait une entreprise : il oriente sa vigilance, sa vivacité et son attention vers de nouveaux buts, de nouvelles ressources disponibles et de nouvelles occasions de profit (Kirzner, 2005). Constamment placé en situation concurrentielle, il est conduit «à surpasser et à devancer les autres dans la découverte de nouvelles occasions de gains » (Dardot et Laval, 2009, p. 221). Doté d'un esprit commercial dans les échanges qu'il opère avec autrui, il exerce constamment ses possibilités d'apprendre, de se corriger et de s'adapter au marché, développant ainsi les dimensions le plus pertinentes de son « capital humain » (Becker, 1964). Par-là, on entend le stock d'acquis dont il hérite, mais aussi ceux qui, via certains investissements opérés dans l'éducation, la santé, les relations, les expériences ou la mobilité, sont à même d'augmenter son potentiel de profits futurs. La gouvernementalité néolibérale et la généralisation de la « forme entreprise » affectent ainsi toutes les sphères de la vie sociale (Foucault, 2004), du travailleur qui engage des dépenses de formation pour obtenir une amélioration future de ses revenus (Becker, 1964), au « consommerçant » (Lemaitre et De Barnier, 2015) qui développe des compétences spécifiques dans la monétisation de ses biens, comme savoir fixer un prix, mettre en scène ses produits et produire de la confiance (Chu et Liao, 2007 ; Garcia-Bardidia, 2014).

\section{II - ...A LA POST-SOCIALITÉ COMME RELATION ETROITE AVEC LES OBJETS}

La généralisation de l'entrepreneurialité n'est pas la seule caractéristique du fonctionnement néolibéral et trois autres facettes lui sont également associées. Knorr Cetina (1997) soutient par exemple que la consécration d'un sujet libre et responsable de lui-même pave la voie de l'individualisme. Le prix à payer de cette liberté coïncide avec l'effondrement de la sphère privée et engendre un fort sentiment d'aliénation. En second lieu, elle suggère que le remplacement d'un certain nombre d'activités humaines par des machines altère le 
social par des formes de savoir et d'expertise distribués sur des objets technologiques (Knorr Cetina, 1997). En conséquence, la machine devient un alter ego, de même que le savoir et l'expertise constituent la base des relations des individus au monde. En troisième lieu, suivant Rheinberger (1992), Knorr Cetina (1997) soutient que nombre de ces activités médiées par des objets ne cessent de changer physiquement de forme et ne se révèlent progressivement à leurs usagers qu'au travers d'apprentissages, d'interactions, d'observations et de réévaluations permanentes (Zwick et Dholakia, 2006). Ils ne sont donc pas seulement des instruments pour l'action ou, comme les marchandises, des signes manipulables sémiotiquement, mais des objets de connaissance. Ces « objets épistémiques » séduisent leurs utilisateurs parce qu'ils stimulent en eux le plaisir d'une exploration extensive des possibilités qu'ils recèlent. Zwick et Dholakia (2006) montrent qu'ils peuvent prendre la forme d'un nouveau logiciel ou de la version augmentée d'un produit technologique, mais aussi d'activités hautement complexes comme les placements boursiers ou la maîtrise technique croissante d'une pratique sportive. Aussi, le développement des plateformes collaboratives pourrait avoir pour effet non seulement de généraliser la figure du consommateurentrepreneur, mais aussi de transformer les relations que ce dernier entretient par et avec ces objets (Knorr Cetina, 1997). C'est cette double perspective que la recherche menée pendant six ans sur la pratique et les pratiquantes du vide-dressing nous conduit maintenant à développer.

\section{Méthodologie de la recherche}

La recherche s'appuie sur une approche multi-méthodes conduite entre mai 2013 à mai 2019 sur la pratique du vide-dressing. Après une étude documentaire qui a fourni une première acculturation au sujet, quinze entretiens longs à caractère ethnographique ont été réalisés auprès de pratiquantes assidues (plus de huit heures de pratique par semaine). Celles-ci ont été recrutées par une méthode en «boule de neige », en essayant de maximiser la variance des profils en termes d'activité professionnelle, de situation familiale et d'âge, profils cependant exclusivement féminins. A ces entretiens, qui totalisent près de 20 heures d'enregistrement et une centaine de pages de retranscriptions, s'ajoutent sept observations non participantes et quatre observations participantes de vide-dressing en face-à-face. Ces observations ont été essentielles pour enrichir les entretiens et dépasser les biais du déclaratif. Enfin, une observation en ligne de différentes plateformes de vide-dressing complète le 
dispositif de collecte. Ainsi, pendant ces 6 années, quatre plateformes (Facebook LilleRoubaix, Vinted, Vestiairecollective et Videdressing) ont été étudiées en continu, générant un corpus de plus de 35 pages de notes, plus de 200 posts et 120 photographies. Certaines des pratiquantes ont été observées sur cette même période, par exemple Charlotte et Estelle sur la page Facebook Vide-dressing Lille-Roubaix, puis lors des vide-dressing organisés dans des lieux publics ou par l'association VioletteSauvage, et enfin lorsqu'elles ont créé une page LesCintrées sur Facebook.

Un processus de codage à visée théorique a ensuite été mené sur l'ensemble des discours. Ce processus de codage itératif a consisté à étiqueter des unités de texte homogènes puis, par un travail de comparaison constante, à dégager et relier les catégories qui ont émergé de l'analyse (Point et Voynnet-Fourboul, 2006). Par exemple, le verbatim suivant : «Il y a des mois où je peux vraiment gagner beaucoup d'argent, les mois d'été avant les vacances, des mois si je vends toutes les semaines, je peux me faire au moins $2000 €$ » (Djamila, 39 ans) a été codé comme «maîtrise de la fonction finance/comptabilité », un sous-thème qui lui-même se relie à d'autres sous-thèmes, comme «maîtrise de la fonction achat», de la fonction «marketing/vente » et de la fonction «logistique » pour former la catégorie « caractère entrepreneurial de la pratique ». C'est ainsi que deux grandes thématiques structurent nos résultats - le caractère entrepreneurial de la pratique du vide-dressing et sa nature d'objet épistémique.

Nous montrons à la suite comment les pratiquantes développent les compétences-clés existant dans une entreprise, avant de mettre au jour en quoi le vide-dressing, via les relations que les pratiquantes développent avec cette activité, constitue un objet épistémique qui transforme les relations avec l'activité et avec les autres pratiquantes.

\section{III - LE VIDE-DRESSING : UNE NOUVELLE PRATIQUE ENTREPRENEURIALE}

En dépit de la dimension ludique de la pratique - «ce qui me plaît c'est l'ambiance, c'est jeune, tu ne te prends pas la tête, c'est superficiel, tu vas parler de mode, de frivolités et trouver LA pièce qui va te plaire » (Djamila, 39 ans) - le côté « sérieux » du jeu marchand apparaît clairement dès les premiers entretiens en 2013. Les pratiquantes «veulent absolument gagner de l'argent » (notes de terrain, 2013) et cherchent à se séparer de certains de leurs vêtements/accessoires en les monétisant. Elles estiment pouvoir en tirer un bénéfice en entretenant un cycle d'achats et de ventes le plus profitable possible, ces possessions 
constituant pour elles un capital hautement valorisable. Nos données montrent également dès les premières observations que les répondantes combinent la pratique du vide-dressing en face-à-face et l'achat/vente en ligne comme l'illustre Charlotte (24 ans) : "Bon moi avant je vendais surtout en vide-dressing (face à face) et je me suis dit qu'il fallait continuer à vendre mes vêtements en dehors. Créer un Vinted (en ligne), c'est se créer un compte et une petite boutique où on met ses photos et ses prix». Cependant, quel que soit le canal choisi, les répondantes doivent être performantes et, comme des professionnelles, développent toutes les compétences-clés (achat, marketing/vente, finance/comptabilité, système d'information et logistique) que l'on trouve dans une entreprise.

La fonction achat consiste à capter les meilleures offres au prix le plus bas, c'est-àdire «avoir la bonne affaire avant tout le monde, être en ligne quand les personnes mettent leurs affaires en vente. Pour cela il faut passer du temps; ça commence le matin dans le tram, pareil le midi, pour voir les nouveautés et le soir à la maison, il faut « shopper » la bonne affaire » (Inès, 22 ans). « Shopper » (ou plutôt choper) les meilleures pièces revient à saisir les marques les plus prisées - Maje, Sandro, Zadig et Voltaire ou plus récemment Sézane -, ce qui implique d'être dotée d'une solide expertise en matière de mode et de connaître le potentiel de revente d'un vêtement, sous peine de rater une aubaine : «J'ai fait une grosse connerie ce weekend. J'ai vu des chaussures Freelance à 10€, je me suis dit : "c'est une marque », mais je ne connaissais pas bien et puis là j'ai vu dans le Vieux Lille, $400 €$ les pompes. Quelle connerie! J'ai dit à mon copain : « là, j'ai loupé quelque chose! »(Estelle, 23 ans).). De fait, il s'agit de spéculer sur les tendances, voire même de parier sur l'évolution des goûts : «En ce moment, c'est la mode des clous, donc j'ai acheté (en vide-dressing) pour revendre. En fait, il faut suivre la mode, même si ce n'est pas du neuf» (Estelle, 23 ans). Les compétences doivent donc être constamment mises à jour, dévoilant la part de calcul, esthétique et commercial, qui oriente l'achat.

La fonction marketing/vente s'exprime quant à elle dans plusieurs registres, le premier étant la mise en scène des produits. Les vendeuses en vide-dressing soignent la présentation de leur stand et/ou de leur vitrine, en adoptant une présentation par taille, par couleur et par marque qui reproduit le merchandising des enseignes : "J'essaye de ranger les pulls, les robes, je fais comme un dressing bien rangé et je mets en avant les pièces tendances. La première pièce sur mon portant, c'est celle qui peut plaire le plus et qui va attirer vers mon stand» (Charlotte, 24 ans). Dans le même esprit, les plateformes comme Vinted, Vestiairecollective ou Videdressing raffinent les fonctionnalités offertes à leurs usagers, qui leur permettent de mettre en valeur leurs produits. Les plateformes publient en 
effet des standards de présentation qui «normalisent une bonne manière » de photographier les vêtements (Illustration 1), conseillant par exemple aux pratiquantes d'intégrer un décor, de soigner l'arrière-plan, et de porter les vêtements au lieu de les présenter à plat.

Illustration 1 : Conseils photo sur www.vinted.fr (mai 2019)

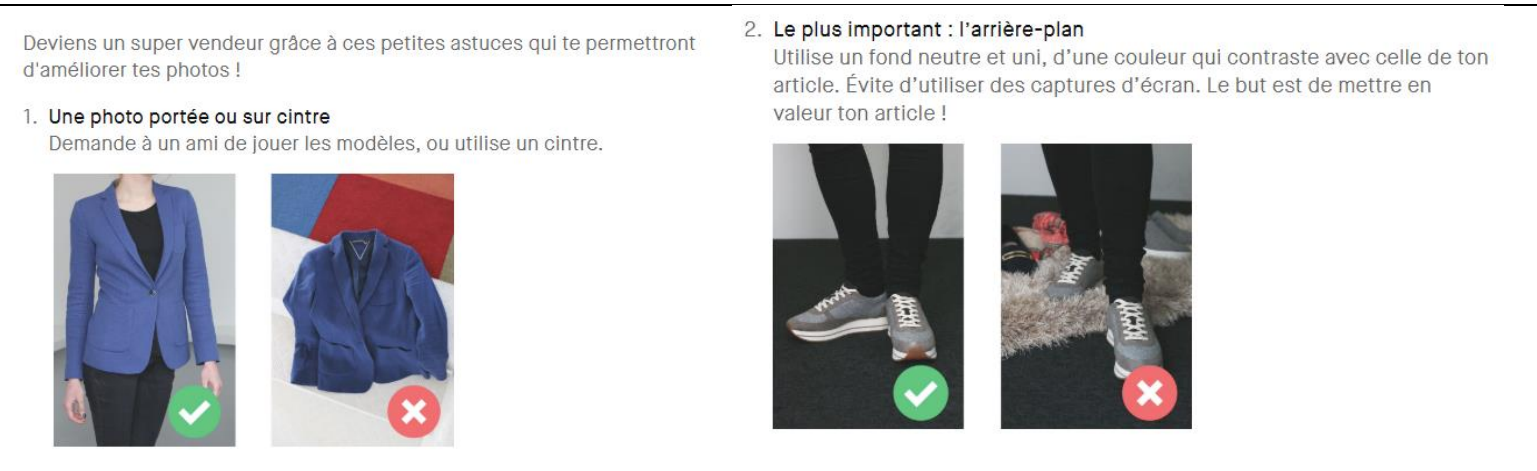

Le deuxième registre d'expertise marketing concerne le prix, pour lequel les pratiquantes reproduisent les stratégies auxquelles l'univers marchand les a acculturées. Les prix sont fixés à l'avance, ce qui encourage les pratiquantes à se former à la cotation des produits d'occasion et à devenir expertes en matière d'estimation des prix. Elles développent aussi une réflexion poussée sur la modulation de leur assortiment, sensible à la saison et à la cible visée. Elles expliquent par exemple que les manteaux doivent être mis en vente avant l'hiver plutôt qu'en plein été et que la bonne période de vente est celle qui suit les soldes où des prix sacrifiés leur ont permis de reconstituer leur stock. De plus, certaines répondantes disent «faire des prix » si la cliente prend plusieurs pièces, pratiquant ainsi une politique de promotion liée à un objectif de fidélisation : «Les dames me remercient, je les fidélise.... Une dame vient tous les 15 jours ou 3 semaines, ça se passe bien, je lui montre ce que je vends » (Stéphanie, 38 ans). Dans ce but, d'autres formes d'expertise sont requises, imposant aux répondantes d'être toujours «aimables, serviables, attentives et souriantes » (note de terrain, 2013). Cependant, les échanges en ligne ne nécessitent pas moins d'attention, à l'instar des stratégies relationnelles mises en œuvre par les marques pour fidéliser leurs clientes : «Moi, quand je fais un envoi, je mets un échantillon de parfum dans le colis, je garde les petits trucs de parfumerie, et moi aussi ça m'arrive de recevoir mon colis avec un petit mot ou autre chose » (Florence, 43 ans). Enfin, la communication constitue un aspect central des compétences en marketing requises par la pratique. Non seulement les plateformes sont en soi des outils de communication - via les «vitrines » qu'elles offrent à leurs clientes -, mais pour les 
évènements physiques, les réseaux sociaux comme Facebook, Instagram et Twitter constituent des vecteurs d'information complémentaires pour des communautés de pratiquantes. Celles-ci peuvent ainsi y annoncer les évènements auxquels elles participent, les pages organisatrices travaillant ensuite le teasing de ces évènements par des messages attrayants (Illustration 2).

Illustration 2 : Annonce Facebook du groupe Les Cintrées (octobre 2016)

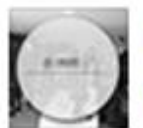

\section{Les cintrées a ajouté 6 photos à l'album Teasing} vêtements Brunch Dressing Basilic Café. 13 octobre

Surveillez bien cet album Teasing qui se remplira jusqu'au 06/11/2016 et qui vous montre une sélection des vêtements de nos différentes vendeuses que vous trouverez lors de notre Brunch Dressing du Basilic Café.

( T34 - $36-38-40)$

\section{[ AUCUN ARTICLE DE CET ALBUM NE SERA VENDU AVANT LA DATE DU VIDE DRESSING POUR DES QUESTIONS DE GESTION ET ORGANISATION - MERCI ]}

La fonction finance/comptabilité n'est pas absente des compétences développées par les pratiquantes et constitue même la finalité première de la pratique puisqu'il s'agit d'investir dans les produits qui permettront de faire le plus de profit possible (ou de perdre le moins possible) : «Même quand t'achètes un manteau 300€, tu sais que tu vas pouvoir le revendre au moins $200 €$ après. Si c'est des pièces très recherchées, tu peux les acheter et ... parfois même ça m'est arrivée de revendre des pièces un an après deux ou trois fois plus cher, parce que c'est recherché »(Djamila, 39 ans). Les pratiquantes développent également des compétences financières en surveillant leur «stock » de vêtements et d'accessoires, dont la rotation est une préoccupation constante. C'est pourquoi elles n'hésitent pas à revendre une pièce portée une seule fois : "Par exemple une robe Maje à 200€, si je l'achète pour une soirée (je l'ai mise une fois), je la revends tout de suite 200€. Je ne gagne pas, mais je ne perds pas! » (Manon, 25 ans). Cet exemple montre que l'achat vestimentaire cesse d'être un acte personnel et fonctionnel - s'habiller - pour devenir une activité économique dont les savoirs et savoir-faire sont entièrement orientés par le calcul. 
La fonction système d'information est également présente. Ainsi, certaines pratiquantes mettent en place une simple gestion papier/crayon de leurs achats et ventes, alors que d'autres utilisent un système informatisé plus sophistiqué qui compile les prix d'entrée, de revente, les dates d'opérations ainsi que la marge réalisée.

Enfin, une bonne maîtrise de la fonction logistique se révèle également centrale dans la pratique du vide-dressing. En amont, celle-ci est conditionnée par des questions spatiales, les pratiquantes étant dans l'obligation d'entreposer de manière soigneuse les objets à vendre : «Je mets cela dans mon ancienne chambre, et chez moi, sous mon lit - comme beaucoup de filles, j'ai déjà remarqué, sous leur lit-c'est une caverne et là où il y a de la place. Bon, c'est vraiment petit chez moi... (en chuchotant), ça prend toute la place... j'achète des meubles pratiques, par exemple, j'ai un petite table de salon, et dedans il y a pleins de sacs, et ils sont fourrés un peu partout » (Léonie, 24 ans). Valoriser son stock implique aussi d'en prendre grand soin. Les vêtements, impeccables, doivent être lavés, repassés et pliés pour ne pas perdre de valeur. En aval, la fonction logistique est marquée par une dimension temporelle car pour ne pas rater une vente, il s'agit de répondre aux clientes le plus rapidement possible et de s'assurer ensuite d'un acheminement sans délai : «Et puis il faut bien suivre. Il faut envoyer en suivi si non c'est : «ahj'ai pas reçu ... ». Enfin ça va, celle qui paie par Paypal, c'est rapide » (Léonie, 24 ans).

De fait, comme l'illustre la Figure 1, la pratique du vide-dressing, en face-à-face comme en ligne, nécessite des compétences gestionnaires multiples - achat, marketing/vente, finance/comptabilité, système d'information et logistique - qui permettent de faire fructifier le capital économique, comme le capital humain de ces consommerçantes (Lemaitre et de Barnier, 2015), 
Figure 1 : Processus entrepreneurial de la pratique du vide-dressing

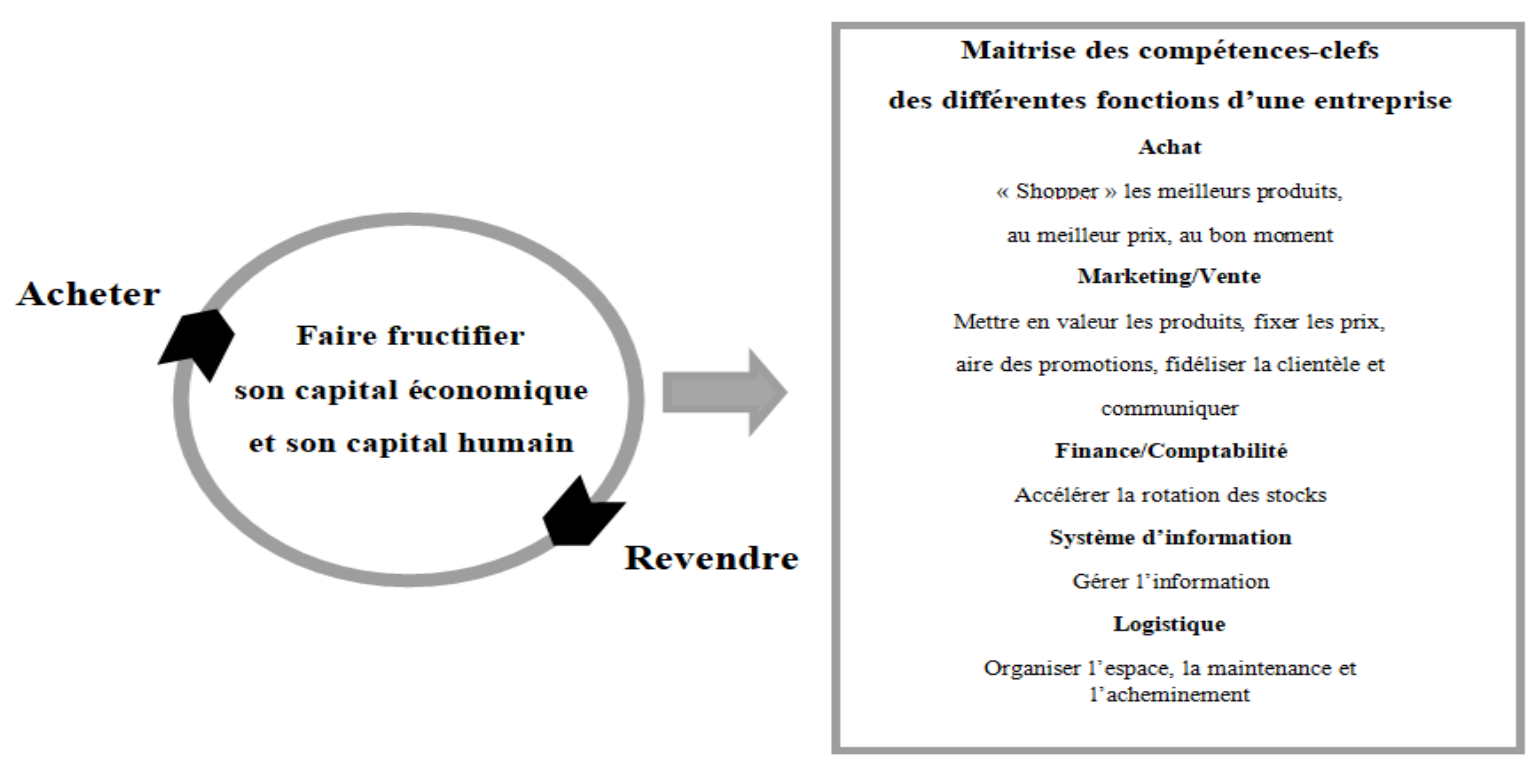

\section{IV - LE VIDE-DRESSING : UN OBJET EPISTEMIQUE}

Dans cette seconde partie des résultats, nous examinons en quoi le vide dressing constitue un objet épistémique au travers des relations que les répondantes entretiennent avec une activité qui les absorbe et nécessite des apprentissages constants. Nous analysons enfin les formes de socialité que ces formes d'échanges participent à transformer.

\section{Une pratique sollicitant une adaptation constante...}

La pratique du vide-dressing ne consiste pas seulement à maîtriser les fonctions-clés de l'entreprise, ni à acquérir certaines compétences que nous avons illustrées. Elle sollicite une adaptation constante du fait que les dispositifs sont en perpétuelle évolution.

\subsection{Des dispositifs (en face-en-face et en ligne) complémentaires}

La pratique du vide-dressing s'est d'abord développée dans des évènements physiques en face-à-face avant que les plateformes ne fluidifient ces échanges en leur donnant une plus longue portée, spatiale et temporelle. Pour autant, les plateformes n'ont pas éclipsé les videdressing physiques. Non seulement les deux formes se complètent, mais elles évoluent chacune à leur manière, obligeant les pratiquantes à s'adapter si elles souhaitent « rester dans la course ». Sur la période d'étude (2013-2019), l'évolution des vide-dressing physiques 
montre que les installations artisanales chez les particuliers, avec des vêtements et des accessoires parfois démodés, voire défraîchis, ont évolué. A partir de 2016, les vide-dressing s'organisent sous la forme de corners semblables à ceux des grands magasins, avec des produits de marque et à la mode. Nos résultats révèlent que l'amateurisme n'est plus de mise. En effet, «le vide-dressing ne veut pas dire friperie et encore moins déchetterie ${ }^{1}$ et nos résultats confirment la montée en qualité des articles présentés. L'état, la composition du vêtement, son descriptif détaillé sont systématiquement requis dans sa présentation, de même que les emballages d'achat et les factures sont désormais fréquemment proposés. La taille et la localisation des évènements aussi se sont modifiées. Au vide-dressing organisé chez soi « et pourtant c'est petit chez moi, on organise, on bouge un peu tout, on met des portiques, je mets tout ce que j'ai à vendre, deux ou trois sacs de chaussures, des accessoires, des vêtements, des robes, et puis je crée un évènement sur Facebook pour inviter des gens, j'invite mes copines et elles, elles invitent leurs amies, c'est la bonne humeur » (Léonie, 24 ans) ont succédé des manifestations d'ampleur, prises en charge par des organisatrices professionnelles. Ainsi, des associations créées et dirigées par des influenceuses du web, comme VioletteSauvage, investissent aujourd'hui des lieux toujours plus grands - le Palais Rameau à Lille ou le Pavillon Baltard à Nogent-sur-Marne - avec pour accroche «Venez shopper le meilleur de la mode à prix canon sur plus de $2700 \mathrm{~m}^{2} \gg$ et l'ambition d'attirer des centaines d'exposantes et d'acheteuses potentiellement candidates à la pratique.

\subsection{Des dispositifs axés sur la mise en concurrence constante des pratiquantes}

Parallèlement, l'apparition des plateformes n'a pas seulement entraîné des modifications dans la professionnalisation des pratiquantes, ce que nous avons montré plus haut, mais aussi dans leur mise en concurrence : «Si tu ne réponds pas t'es mal notée - tu as des appréciations après tes envois -, et si tu as des mauvaises appréciations, alors on n'achète pas dans ton dressing » (Léonie, 24 ans). Les systèmes de notation illustrent de fait le caractère « auto-éducateur et auto-disciplinaire » du système (Dardot et Laval, 2009, p. 226) qui engage les pratiquantes dans une lutte concurrentielle semblable à celle des professionnels marchands. Comme l'illustre Charlotte (24 ans), organisatrice de vide-dressing en face-àface : «Il y a des filles qui donnent beaucoup d'importance à " je suis une bonne vendeuse » ... clairement si cela se passe mal, la vendeuse a une mauvaise note et ça va lui plomber son profil, après pour les ventes elle va être freinée. Il y a un côté pro finalement ». De fait, l'organisation de la compétition ne se limite pas aux plateformes et s'exprime tout autant dans

\footnotetext{
${ }^{1}$ http://madame.lefigaro.fr/style/vide-dressing-5-conseils-pour-bien-vendre-221216-128738
} 
les échanges en face-à-face: "Voici un fichier Excel et toutes les vendeuses que j'ai sélectionnées. Moi, je fonctionne avec des couleurs. Bon celle-là, elle est verte : elle fait partie des bonnes vendeuses. Elle joue le jeu, on peut avoir confiance. Au vert on passe, au rouge, on arrête. On arrête avec celle qui n'a pas souri de tout l'événement, qui n'est pas à l'heure, qui a laissé son stand n'importe comment, ou qui part avant la fin, ça c'est gênant ! » (Charlotte, 24 ans). Le fait que de nombreux forums ou sites de conseil se multiplient pour aider les novices à «réussir» leur vide-dressing témoignent également de la professionnalisation constante de la pratique et de la nécessité, pour celles qui souhaitent s'y engager, de maitriser des règles qui ne cessent de s'affiner (bien photographier l'objet, choisir les produits les plus tendances, savoir organiser stands et vitrines, bien se comporter avec les clientes, etc.). C'est en effet au cours des interactions, des observations et des leçons tirées de l'activité, en ligne comme en face-à-face, que les pratiquantes progressent, sans cesser d'adapter leurs efforts aux exigences que la pratique requiert. En ce sens, le vide-dressing constitue un objet épistémique, capable de stimuler celles qui cherchent à se surpasser et à surclasser les autres dans la compétition marchande (Dardot et Laval, 2009).

Figure 2 : La pratique du vide-dressing un objet épistémique

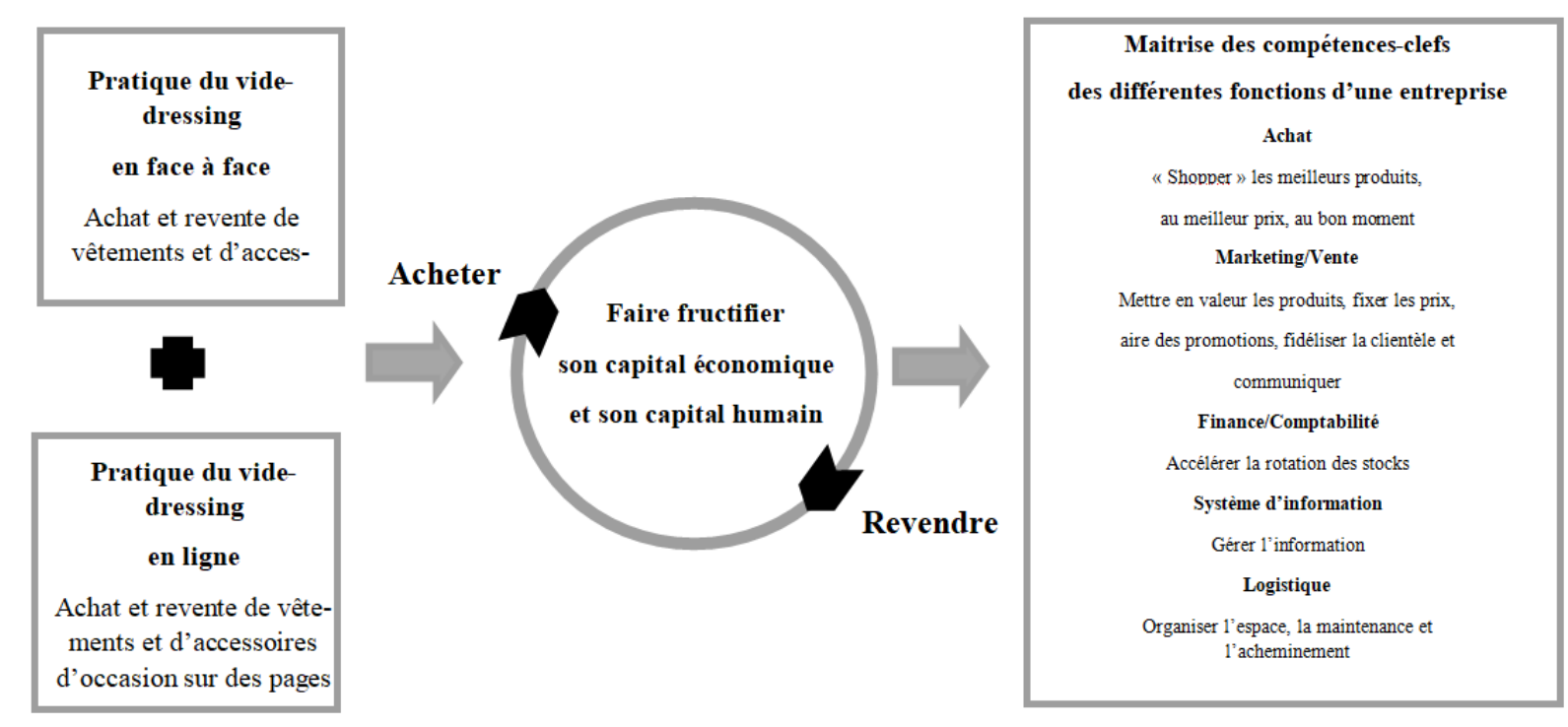

\section{2. ...et qui engendrent des formes de post-socialité.}

Si la pratique apporte de nouvelles compétences, un réel plaisir et une forme 
d'épanouissement personnel, la place qu'elle occupe dans la vie des répondantes les absorbe au point qu'elle tend à prendre le pas sur d'autres relations sociales. Se pose alors des questions quant aux formes de socialité que cette pratique développe et transforme. Dans certains cas, le rapport avec l'activité peut s'avérer invasif et quasi-exclusif : «Je suis en train de chercher une maison, mais plutôt que de chercher, je suis sur un vide-dressing. Et mon copain me demande: «tu fais quoi ?»... «Rien, rien». S'il savait, je me ferais «tuer»!!! J'y suis trop. Le midi j'ai 2 h et au lieu d'être avec mes collègues, je mange en 20 minutes pour retourner voir mes messages ... ça devient ... comme une drogue !!! On a envie d'aller voir » (Manon, 25 ans). Les repas avec les collègues, voire la vie de couple, sont parfois délaissés au profit de cet engagement intense dans la pratique. Les données révèlent alors une relation singulière qui se noue avec l'activité, à la fois en termes d'appétence «C'est comme jouer à la marchande » (Stéphanie, 38 ans), «C'est cool, je vais faire du business !»(Estelle, 23 ans) -, mais aussi d'intensité et de fréquence. Certaines répondantes sont, en effet, immergées dans une activité qui devient centrale dans leur vie et dont l'entourage pointe parfois l'exagération : «Mon copain il m'a dit : «non mais ça va pas !!! C'est des vêtements usagés !!! ». Donc je le comprends, oui maintenant je me suis calmée. Mais, c'est comme un divertissement, c'est un plaisir, c'est une drogue, il faut être les premières à voir ça!!! »(Ophélie, 23 ans). L'engagement dans la pratique peut s'avérer excessif au point que certaines répondantes la comparent à une addiction. Bon nombre d'entre elles participent en effet à plus de dix vide-dressing par an et se connectent plus de dix fois par jour à leur compte Facebook ou sur les plateformes, certaines avouant même être connectées en permanence. Le rapport à cette activité largement chronophage est renforcé par la place qu'y tiennent les outils numériques. Les plateformes renvoient aux pratiquantes toutes les informations qu'elles cherchent à contrôler, telles que leur délai de réponse et la date de leur dernière visite. Cet état d'hyper-information, associé à des dispositifs de notation par les pairs, renforce une relation de plus en plus intense avec les outils qui, en liant fréquence d'interaction et performance, les engagent toujours plus dans une maîtrise croissante de la pratique.

En occupant une place essentielle dans leur vie quotidienne, cette pratique modifie la nature des relations privées, mais aussi des échanges marchands. Si les premières observations menées en 2013 montraient que ces échanges reposaient majoritairement sur des transactions de gré à gré et en coprésence, la montée en puissance des plateformes numériques depuis 2016 révèle une modification sensible. Ainsi c'est en 2016, que des messages automatisés du type «faire une offre », «offre refusée » «offre acceptée » font leur 
apparition sur les plateformes, limitant les conversations entre usagers. En accélérant les transactions «en quelques clics » et sans interaction physique, les plateformes transforment les liens : elles délèguent aux outils une médiation technique qui se substitue aux échanges directs avec les pairs, tels qu'ils préexistaient. De manière concomitante, d'autres indices attestent à la même période du caractère plus «industriel» de l'activité dans le monde physique. Le concept de vide-dressing « géant» rompt avec les codes de l'amusement, de la proximité et de l'artisanat qui prévalaient antérieurement dans les évènements organisés par des particuliers à leur domicile. Certes l'ambiance reste conviviale - en apparence -, mais l'objectif désormais assumé est de «faire des affaires » et pour cela les pratiquantes sont triées sur le volet. Les organisateurs reconnaissent en effet être « sélectifs » et chercher à dénicher «les meilleurs dressings», car leur priorité est de «satisfaire acheteurs ET vendeurs » en évinçant celles qui vendraient «des pièces démodées ».

Nos observations témoignent également de négociations plus rudes entre acheteuses et vendeuses. Il est aujourd'hui moins question de se retrouver ou de s'amuser entre copines que d'échanger entre expertes de l'achat ou de la vente détenant un savoir-faire gestionnaire et démontrant une supériorité dans la maîtrise des règles du jeu.

Figure 3 : Les formes de post-socialité engendrées par la pratique du vide-dressing

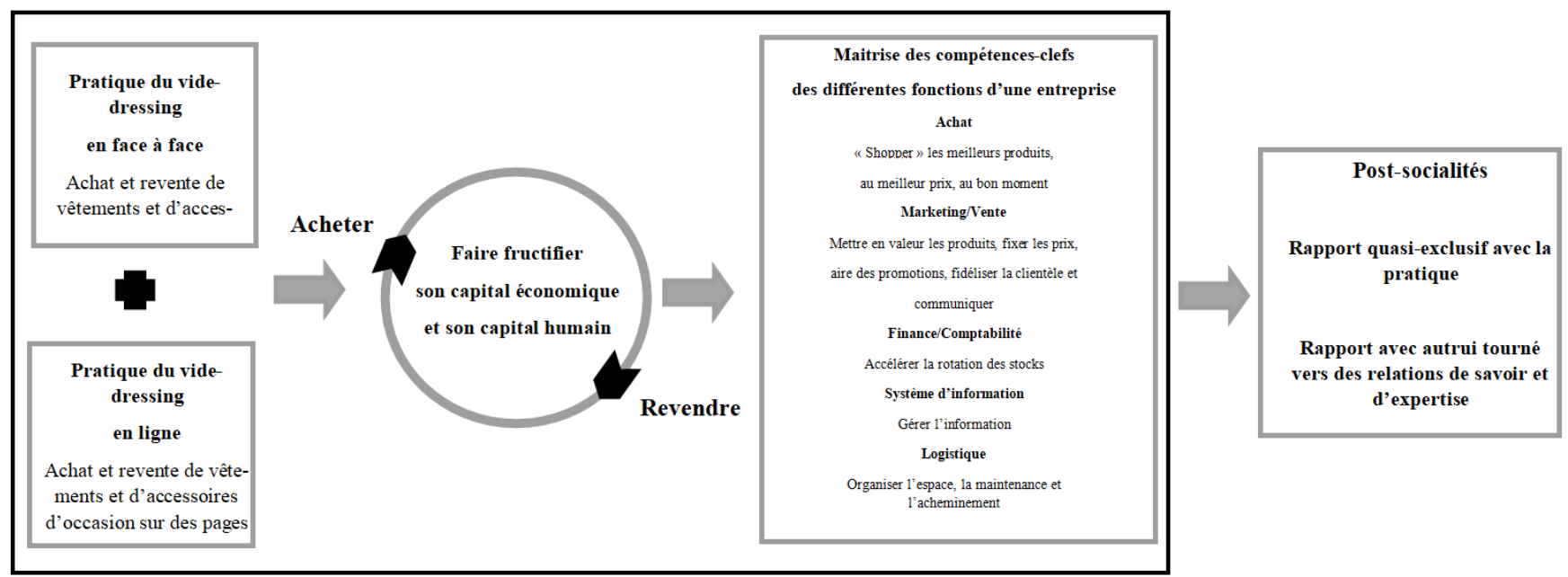

\section{V - DISCUSSION, LIMITES ET VOIES DE RECHERCHE}

Cette recherche conduite pendant six années dans le contexte du vide-dressing éclaire sous un jour nouveau les formes d'entrepreunarialité et de post-socialité qui découlent de la 
consommation dite collaborative. Alors même que ces systèmes d'échanges se paraient de promesses sociales, voire environnementales (Borel, Massé et Demailly, 2015 ; Botsman et Rogers, 2011 ; Robert, Binninger et Ourahmoune, 2014), seule la dimension économique semble les animer, faisant écho à l'existence d'un type de consommateur-commerçant - le « consommerçant » (Lemaitre et De Barnier, 2015) -, intéressé par la vente et pas seulement par l'achat. Par ailleurs, cette recherche complète les travaux existants sur la consommation collaborative et les formes marchandes d'achat/vente en ligne (Decrop, 2017 ; Denegri-Knott et Molesworth 2009 ; Garcia-Bardidia, 2014 ; Trespeuch et al., 2019) en dévoilant deux facettes essentielles de la gouvernementalité à l'œuvre dans les pratiques collaboratives.

En premier lieu, elle montre que ces échanges entre particuliers relèvent de la «forme entreprise » (Foucault, 2004), au-delà de la simple orientation marchande des pratiques (Herbert et Collin-Lachaud, 2016) et développent des formes de compétences variées et dirigées, comme dans la « forme entreprise », vers la maximisation des capitaux économiques et humains. En effet, cette pratique ne banalise pas seulement le fait de tirer profit de ses biens personnels - qui, antérieurement, soit restaient dans la sphère privée, soit étaient transmis, troqués ou donnés (Albinsson et Yasanthi Perera, 2012) -, mais elle met au jour l'acquisition de compétences-clés, chez des individus réputés non professionnels. Cette montée en compétences est toutefois peu discutée dans les travaux sur la consommation collaborative, en comparaison de l'orientation marchande/non marchande (Daumas, 2018) et du degré de socialisation qu'elles engendrent (Herbert et Collin-Lachaud, 2016 ; Trespeuch et al., 2019). Ainsi, le savoir vendre, acheter, négocier, investir, fidéliser, communiquer, stocker et gérer peut autant s'appliquer aux voitures, qu'aux logements ou à d'autres objets comme le vêtement que l'on prêtait auparavant dans des cercles d'interconnaissance, mais qu'on loue ou vend désormais à des inconnus par l'intermédiaire de plateformes ou de vide-dressing « géants » (Borel, Roux et Demailly, 2015).

En second lieu, la recherche montre que la pratique du vide-dressing s'accompagne d'interactions physiques qui tendent à se raréfier ou qui, quand elles perdurent, s'expriment sur un mode concurrentiel exacerbé par les acteurs qui organisent ces échanges. Ces résultats révèlent combien cette activité - physique ou en ligne - infléchit les relations humaines vers une post-socialité dans laquelle les systèmes techniques captent et dirigent l'attention prioritairement vers les outils et les dispositifs (Knorr Cetina, 1997). Au final, les pratiques de vide-dressing focalisent l'attention de leurs usagers sur la formation à une compétition toujours mieux maîtrisée grâce au déploiement de techniques de mise en marché et des dispositifs de conseil, de notation et d'aide à la valuation des biens comme des personnes. 
Objet épistémique contraignant ses usagers à l'adaptation constante de leurs savoirs, la pratique du vide-dressing est également à la source d'une post-socialité produite par ces nouveaux dispositifs.

Cette prééminence de la «forme entreprise » dans la monétisation croissante des biens personnels, le développement du capital économique et humain qui l'accompagne, comme la transformation des relations à soi, aux objets et aux autres, ouvrent plusieurs fronts de réflexion. Tout d'abord, et contrairement à certaines promesses (Botsman et Rogers, 2011), ces pratiques ne contribuent pas fondamentalement à un ralentissement de la consommation, bien au contraire (Parguel, Lunardo et Benoit-Moreau, 2017). La possibilité de revendre rapidement et facilement ses biens stimule un capitalisme, de plateformes mais pas seulement, qui est largement décrié (Benavent, 2016). La pratique du vide-dressing agit également sur le marché neuf et le renouvellement accéléré des modes, mais aussi sur le marché de l'occasion où son impact est inhabituel. En effet, le travail de sélection, de choix et de remise en marché de certains biens qui en sont extraits par le jeu des pratiquantes opère une requalification, une singularisation, voire une revalorisation des produits de seconde main. La pratique du vide-dressing nourrit de facto le marché de l'occasion qui, loin d'alimenter une offre décotée, participe à créer et soutenir la valeur de certaines marques et à faire de chaque pièce une «singularité » (Karpik, 2007). Dans ces processus, les plateformes mais aussi les évènements géants gérés par de nouveaux intermédiaires, jouent un rôle déterminant en créant simultanément les compétences et les pratiquantes qu'elles s'emploient à outiller, former et professionnaliser, en standardisant les « bonnes manières de faire », en simplifiant les opérations d'échange et en accélérant les gestes de mise en marché. Il en résulte une série d'effets plus ou moins contradictoires. Ainsi, d'un côté ces intermédiaires sociotechniques apportent une valeur ajoutée aux modalités de développement du capital humain des individus. Ceux-ci, de consommateurs passifs et destructeurs de valeur, deviennent des individus actifs, rompus à la fourniture de biens et services et à la production de valeur, modifiant ainsi les caractéristiques de la « consommation conventionnelle » (Ertz, Durif et Arcand, 2016). Par ailleurs, alors que les socialités primaires d'interconnaissance (Borel, 2011) sont souvent porteuses de tensions, sont chronophages et exigent un fort engagement relationnel, les dispositifs sociotechniques - plateformes, applications mobiles, voire organisations d'évènements à grande échelle - suppriment les relations de face-à-face et modifient la grammaire des échanges en déléguant massivement aux « outils » le cadrage des transactions. Ces outils produisent de nouvelles socialités « tertiaires »-par opposition aux socialités primaires de l'interconnaissance et aux socialités secondaires du contrat (Borel, 
2011) -, qui règlent les relations en les rendant plus agiles, plus rapides et plus fluides. Inversement, et nos résultats le montrent, l'efficience recherchée dans la pratique tend à effacer les formes de relations qui tramaient les échanges de gré à gré, plus approximatifs et artisanaux, comme elle parasite parfois les relations domestiques environnantes. On pourrait dire, en renversant la formule de Cova (2004), que «le bien importe plus que le lien » et transforme de fait les relations sans calcul et sans recherche intensive du profit.

Au final, ce travail n'est pas exempt de limites mais il ouvre des perspectives sur des phénomènes en émergence. Dans d'autres domaines d'activité comme le travail, les rencontres ou la santé, il serait intéressant de prolonger cette recherche pour étudier si les formes de post-socialité aboutissent par exemple à cautionner la suppression des caissières et des guichetiers dans la relation de service, à modifier les conventions de la quête amoureuse sur les sites de rencontre (Kessous, 2011) ou à transformer la relation patient/médecin qu'à l'époque de la désertification médicale on tend à rabattre sur une interaction à distance et avec des machines. L'étude de ces différents domaines permettraient également d'aborder des terrains moins exclusivement genrés, cette recherche n'impliquant que des femmes. Il serait en outre intéressant d'observer d'autres pratiques d'échanges entre particuliers portant sur des biens d'investissements lourds (co-voiturage ou location de biens immobiliers) pour examiner si les mêmes mécanismes prévalent ou lesquels les différencient du vide-dressing. Des pratiquantes ayant renoncé au vide-dressing mériteraient également d'être interrogées pour comprendre les raisons qui conduisent à d'autres modalités d'emprunt que sont, à la manière des livres, des «bibliothèques de vêtements » (Pederson et Netter, 2015), mais également à l'abandon de cette activité pour des raisons pratiques (manque de temps), cognitives (difficultés de maniement des outils numériques), voire idéologiques ou critiques de l'exploitation marchande (Abdelnour, 2016; Dujarier, 2008). Enfin, une approche comparative des formes de socialités fournirait un prolongement essentiel à cette recherche en analysant les effets cumulatifs de ces pratiques sur des populations jeunes, comparés à des populations plus âgées, et décrivant les transformations que les dispositifs sociotechniques opèrent progressivement dans les relations entretenues dans le temps avec les objets et avec autrui.

\section{Références}

Abdelnour S. (2016), Moi, petite entreprise. Impacts individuels et collectifs de la diffusion de l'auto-entrepreneuriat, Regards croisés sur l'économie, vol. 2, n 19, 192-203.

Acquier A., Carbone V. et Massé D. (2017), L'économie collaborative, source d'innovation 
sociétale?, In La consommation collaborative. Enjeux et défis de la nouvelle société du partage, A. Decrop (coord.), de Boeck Supérieur, Louvain-la-Neuve, 243-268.

Albinsson P.A. et Yasanthi Perera B. (2012), Alternative marketplaces in the 21st century: Building community through sharing events, Journal of Consumer Behaviour, vol. 11, $n^{\circ} 4,303-315$.

Bauhain-Roux D. et Guiot D. (2001), Le développement du marché de l'occasion: Caractéristiques et Enjeux pour le marché du neuf, Décisions Marketing, n 24, 25-35.

Becker G. (1964), Human capital. A theoretical and empirical analysis with special reference to education, The University of Chicago Press, Chicago.

Belk R. (2010), Sharing, Journal of Consumer Research, vol. 36, ${ }^{\circ}$ 5, 715-734.

Benavent C. (2016), Plateformes. Sites collaboratifs, marketplaces, réseaux sociaux... Comment ils influencent nos choix, FYP Editions, Paris.

Borel S. (2011), Les liaisons numériques. Dangereuses ou vertueuses ?, Revue du MAUSS, vol. $38, n^{\circ} 2,349-368$.

Borel S., Massé D. et Demailly D., (2015), L'économie collaborative, entre utopie et big business, Revue Esprit, vol. 416, $\mathrm{n}^{\circ}$ 7, 9-18.

Borel S., Guillard V. et Roux D. (2017), Echanger entre étrangers : calcul ou partage ?, In A. Decrop (coord.), La consommation collaborative. Enjeux et défis de la nouvelle société du partage, DeBoeck Supérieur, Louvain-la-Neuve, 85-108.

Botsman R. et Rogers R. (2011), What's mine is yours: How collaborative consumption is changing the way we live 2de Edition, Harper Collins, Londres.

Chu H. et Liao S. (2007), Exploring consumer resale behavior in C2C online auctions: Taxonomy and influences on consumer decisions, Academy of Marketing Science Review, vol. $11, \mathrm{n}^{\circ} 3,1-48$.

Clarke, A. (2000), Mother swapping: the trafficking of nearly new children's wear. In Jackson, P., Lowe, M., Miller, D. et al. (eds), Commercial cultures: Economies, practices, spaces, Oxford and New York: Berg, 85-100.

Cova B. (2004), Au-delà du marché. Quand le lien importe plus que le bien, L'Harmattan, Paris.

Cova B. et Cova V. (2009), Les figures du nouveau consommateur : une genèse de la gouvernementalité du consommateur, Recherche et Applications en Marketing, vol. 24, $\mathrm{n}^{\circ}$ 3, 81-100.

Dardot P. et Laval C. (2009). La nouvelle raison du Monde. Essai sur la société néolibérale, La Découverte, Paris.

Daumas J.-C. (2018), La révolution matérielle. Une histoire de la consommation (France XIXe-XXIe siècle), Flammarion, Paris.

Decrop A. (2017), La consommation collaborative : Enjeux et défis de la nouvelle société du partage, De Boeck Supérieur, Louvain-la-Neuve.

Denegri-Knott J. et Molesworth M. (2009), 'I'll sell this and I'll buy them that': eBay and the management of possessions as stock, Journal of Consumer Behaviour, Vol. 8, $\mathrm{n}^{\circ}$ 6, 305 315.

Dujarier M-A. (2008), Le travail du consommateur de McDo à eBay: comment nous coproduisons ce que nous achetons, La Découverte, Paris.

Ertz, M., Durif F. at Aracand M., (2016), Collaborative consumption: conceptual snapshot at a buzzword, Journal of Entrepreneurship Education, 19, 2.

Foucault M. (2004), Naissance de la biopolitique. Cours au Collège de France, 1978-1979, Gallimard-Seuil, Paris.

Garcia-Bardidia R. (2014), Se débarrasser d'objets sur leboncoin.fr. Une pratique entre don et marché ?, Revue du MAUSS, vol. 44, n² 2, 271-285.

Hamari J., Sjöklint M. et Ukkonen A. (2015), The Sharing Economy: Why People Participate 
in Collaborative Consumption, Journal of the Association for Information Science and Technology, vol. 67, $\mathrm{n}^{\circ}$ 9, 2047-2059.

Herbert M. et Collin-Lachaud I. (2016), Pratiques collaboratives et habitus consumériste : une analyse des mécanismes transformatifs de la consommation collaborative, Recherche et Applications en Marketing, vol. 32, $\mathrm{n}^{\circ}$ 1, 42-62.

Karpik L. (2007), L'économie des singularités, Gallimard, Paris.

Kessous E. (2011), L'amour en projet. Internet et les conventions de la rencontre amoureuse, Réseaux, vol.166, $\mathrm{n}^{\circ} 2,191-223$.

Kirzner I. (2005), Concurrence et esprit d'entreprise, Economica, Paris.

Knorr Cetina K. (1997), Sociality with objects. Social Relations in Postsocial Knowledge Societies, Theory, Culture and Society, vol. 14, n 4, 1-43.

Lemaître N. et (de) Barnier V. (2015), Quand le consommateur devient commerçant: motivations, production d'expérience et perspectives, Décisions Marketing, $\mathrm{n}^{\circ}$ 78, 11-28.

Lurie A.(1981), The Language of Clothes, New York: Vintage Books.

Ozanne L. et Ballantine P. (2010), Sharing as a form of anti-consumption? An examination of toy library users, Journal of Consumer Behaviour, 9, 485-498.

Parguel B., Lunardo R. et Benoit-Moreau F. (2017), Sustainability of the sharing economy in question: When second-hand peer-to-peer platforms stimulate indulgent consumption, Technological Forecasting and Social Change, 125, 48-57.

Pedersen E. R. et Netter S. (2015), Collaborative consumption: business model opportunities and barriers for fashion libraries, Journal of Fashion Marketing and Management, 19 (3), 258-273. 
Pike, H. (2016), Will the 'Sharing Economy' Work For Fashion? Retrieved May 13, 2019 from Business of Fashion.

https://www.businessoffashion.com/articles/fashion-tech/will-the-sharing-economyworkfor-fashion-rent-the-runway-rental

Point S. et Voynnet-Fourboul V. (2006), Le codage a visée théorique, Recherche et Applications en Marketing, vol. 21, $\mathrm{n}^{\circ}$ 4, 61-78.

Rheinberger H.-J. (1992), Experiment, Difference and Writing: I. Tracing Protein Synthesis, Studies in the History and Philosophy of Science, vol. 23, $\mathrm{n}^{\circ} 2,305-331$.

Ritzer G. et Jurgenson N. (2010), Production, Consumption, Prosumption, Journal of Consumer Culture, vol. 10, $\mathrm{n}^{\circ} 1,13-36$.

Robert I., Binninger A-S. et Ourahmoune N. (2014), La consummation collaborative, le versant encore équivoque de l'économie de la fonctionnalité, Développement durable et territoires, Vol. 5, $\mathrm{n}^{\circ} 1$, Février. Version en ligne.

Roux D. et Korchia,M. (2006), I am what I wear?: An exploratory study of symbolic meanings associated with secondhand clothing, Advances in Consumer Research, 33, 1-7.

Schumpeter J. A. (1990 [1947]), Capitalisme, socialisme et démocratie, Payot, Paris.

Stehr N. (1994), Knowledge Societies, Sage Publication, London.

Toffler A. (1980), The rise of the prosumer: The third wave, William Morrow and Co, New York.

Trespeuch M., Beuscart J.-S., Pharabod A.-S. et al. (2019), Échanger entre particuliers : construction et euphémisation du lien marchand à l'heure numérique, Revue Française de Socio-Économie, Vol. 22, $\mathrm{n}^{\circ} 1,125-150$.

Von Mises L. (1985[1949]), Human action. A treatise on economics, Yale University Press New Haven.

Zwick D. et Dholakia N. (2006), The Epistemic Consumption Object and Postsocial Consumption: Expanding Consumer-Object Theory in Consumer Research, Consumption, Markets and Culture, vol. 9, $\mathrm{n}^{\circ} 1,17-43$. 